\title{
Deforming arthropathy or lupus and rhupus hands in systemic lupus erythematosus
}

\author{
R M van Vugt, R H W M Derksen, L Kater, J W J Bijlsma
}

\begin{abstract}
Objective-Although deforming arthropathy in systemic lupus erythematosus (SLE) is characterised by a number of manifestations, definitive criteria for the different forms have not yet been established. To define deforming arthropathy and its different types a study was undertaken of 176 SLE patients.

Methods-Using as criterion any deviation from any of the metacarpus finger axes 17 patients (16 women, one man) were identified with clinical deforming arthropathy. These patients were evaluated according to a standardised protocol that covered all known characteristics of deforming arthropathy. By means of "Jaccoud's arthropathy index" three different forms were identified.
\end{abstract}

Results-Three patients had an erosive form of deforming arthropathy (or rhupus hand) such as those seen in frank rheumatoid arthritis (RA), eight patients were identified as having Jaccoud's arthropathy (or lupus hand), and the remaining six patients had mild deforming arthropathy. Jaccoud's arthropathy is characterised by severe deformation of the hands (ulnar deviation, swan neck deformities, and $Z$ deformity of the thumb) and feet with multiple non-erosive subluxations, mild aching and little or no evidence of synovitis. All patients, but one, fulfilled just four criteria of the ACR classification and joint symptoms were always found to precede the diagnosis of SLE. Furthermore a remarkable association of Jaccoud's arthropathy with fetal loss, thrombosisboth venous and arterial-and the presence of antiphospholipid antibodies was found.

Conclusions-These data suggest that Jaccoud's arthropathy represents a subset of SLE. Subdivision of deforming arthropathy into several clinical forms can facilitate the clinical management of this disorder.

(Ann Rheum Dis 1998;57:540-544)

Department of Rheumatology and Clinical Immunology, University Hospital Utrecht, PO Box 85500,3508 GA

Utrecht, the

Netherlands

Correspondence to: Dr van Vugt.

Accepted for publication 14 July 1998

Involvement of the joints in systemic lupus erythematosus (SLE) (lupus arthropathy) is one of the earliest and most common manifestations of this multisystemic disease. The degree of involvement may range from minor arthralgia to severe deforming arthritis. ${ }^{12}$

Pain and stiffness are more common than objective abnormalities and the synovitis of SLE is generally transient, migratory, and reversible. ${ }^{3}$ Occasionally it may take a more chronic course, leading to joint deformity that affects predominantly the finger joints, the wrists, and the knees. ${ }^{4}$ In the extreme case the hand deformities form a typical picture of ulnar deviation and subluxations that together resemble that of rheumatoid arthritis (RA). ${ }^{5}$ Bywaters was the first to point out the similarity between the deforming arthritis of SLE and that reported in 1869 by Jaccoud for recurrent rheumatic fever. ${ }^{56}$ Although Jaccoud's arthropathy has been described together with a variety of disorders, no definitive criteria for diagnosis have been published so far. ${ }^{7}$

Other forms of deforming arthropathy have not yet been described in detail and the question is whether the erosive form represents the coexistence of SLE and RA. ${ }^{3}$ The purpose of this study was to describe deforming arthropathy, identify the different forms and correlate these forms with the various clinical and laboratory features of the disease.

\section{Methods}

In accordance with Alarcon-Segovia et $a l^{5}$ we considered any deviation, assessed with an angle goniometer correctable or not, from any of the metacarpus finger axes as deforming arthropathy in SLE. Using this criterion we identified 17 patients with clinical deforming arthropathy of the hands in our total group of 176 patients (160 female and 16 male) who fulfilled the ACR revised criteria for the classification of SLE. The study group consisted of 16 women and one man with a mean age at onset of disease of 20.8 years (range 13-40) and a mean disease duration of 15.6 years (range 4-35).

These patients were evaluated according to a protocol that covered all known characteristics of deforming arthropathy. ${ }^{3-7}$ Physical examination included a detailed standardised examination of the hands and feet. The following items were evaluated in each case: signs of arthritis, ulnar deviation of fingers, metacarpophalangeal subluxation, swan neck deformities of the fingers, $\mathrm{Z}$ deformity of the thumb, boutonnière

Table 1 Index for establishing the diagnosis of Faccoud's arthropathy. Faccoud's arthropathy was considered present if the score exceeded 5 points

\begin{tabular}{lll}
\hline Faccoud's arthropathy Index & $\begin{array}{l}\text { Number of } \\
\text { affected fingers }\end{array}$ & Points assigned \\
\hline Ulnar drift $(>20)$ & $1-4$ & 2 \\
Swan neck deformities & $5-8$ & 3 \\
& $1-4$ & 2 \\
Boutonniere deformities & $5-8$ & 3 \\
& $1-4$ & 1 \\
Z deformity & $5-8$ & 2 \\
& 1 & 2 \\
& 2 & 3 \\
\hline
\end{tabular}




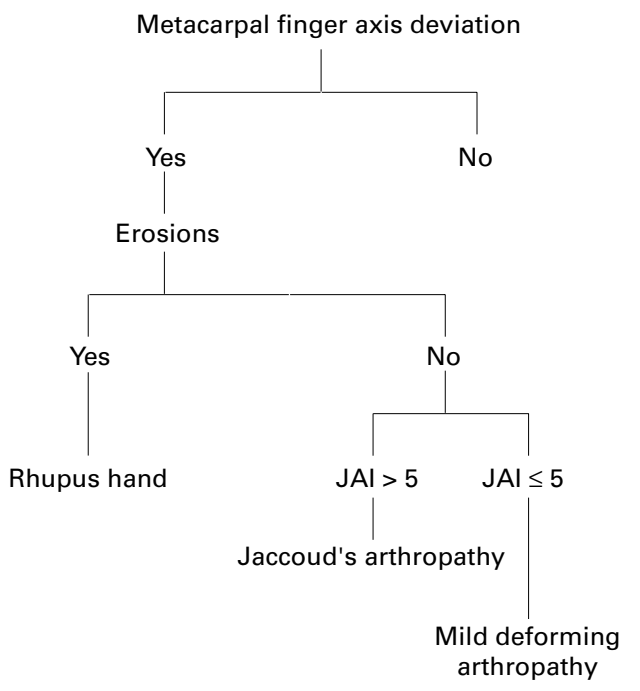

Figure 1 Flow diagram by which the diagnosis of the three different forms of deforming arthropathy is reached.

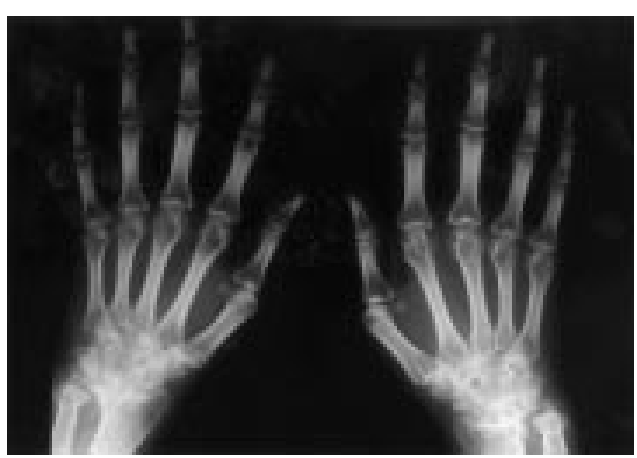

Figure 2 Radiograph of erosive arthropathy (rhupus hand) showing joint space narrowing and rheumatoid-like bone erosions in the intercarpal and radiocarpal joints. Mild erosive damage of some MCP joints.

deformities, grip strength (assessed with a Martin vigorimeter and calculated as the mean of three measurements), hallux valgus and hammer toes.

Patients were asked about Raynaud's phenomenon, pain, and functional impairment of the hands and problems with the feet (pain or

Table 2 Comparison of characteristics of the three types of deforming arthropathy

\begin{tabular}{|c|c|c|c|}
\hline Patient characteristics & $\begin{array}{l}\text { faccoud's } \\
\text { arthropathy } \\
(n=8)\end{array}$ & $\begin{array}{l}\text { Erosive } \\
\text { arthropathy } \\
(n=3)\end{array}$ & $\begin{array}{l}\text { Mild deforming } \\
\text { arthropathy } \\
(n=6)\end{array}$ \\
\hline Age at onset (range) & $23.3(17-39)$ & $19.6(14-24)$ & $23.8(19-25)$ \\
\hline Disease duration (range) & $23.7(11-39)$ & $10.6(4-15)$ & $11.6(8-17)$ \\
\hline Functional impairment & $3 / 8$ & $2 / 3$ & $3 / 6$ \\
\hline Pain & $1 / 8$ & $2 / 3$ & $4 / 6$ \\
\hline Feet involved & $8 / 8$ & $1 / 3$ & 0 \\
\hline Hypermobility & $1 / 8$ & 0 & $1 / 6$ \\
\hline Synovitis wrist & 0 & $3 / 3$ & 0 \\
\hline Synovitis fingers & 0 & $1 / 3$ & $4 / 6$ \\
\hline Pain at pressure & 0 & $3 / 3$ & $2 / 6$ \\
\hline \multicolumn{4}{|l|}{ Swan neck deformity } \\
\hline $1-4$ fingers & $3 / 8$ & $1 / 3$ & $3 / 6$ \\
\hline $5-8$ fingers & $5 / 8$ & $1 / 3$ & 0 \\
\hline \multicolumn{4}{|l|}{ Ulnar deviation } \\
\hline $1-4$ fingers & $1 / 8$ & $1 / 3$ & $5 / 6$ \\
\hline $5-8$ fingers & $7 / 8$ & $2 / 3$ & 0 \\
\hline \multicolumn{4}{|l|}{ Boutonniere deformity } \\
\hline $1-4$ fingers & $2 / 8$ & $1 / 3$ & 0 \\
\hline $5-8$ fingers & 0 & 0 & 0 \\
\hline $\mathrm{Z}$ deformity one or both thumbs & $8 / 8$ & $2 / 3$ & $2 / 6$ \\
\hline Mean grip strength $(0-120 \mathrm{kpa})$ & 31 & 33 & 44 \\
\hline Hallux valgus & $4 / 8$ & $1 / 3$ & $1 / 6$ \\
\hline Metatarsal subluxation & $3 / 8$ & $1 / 3$ & 0 \\
\hline Hammer toes & $5 / 8$ & 0 & $1 / 6$ \\
\hline
\end{tabular}

deformities necessitating semi-orthopaedic shoes or surgery). These items were scored as "positive" or "negative".

Previous history with special attention to the interval between first joint symptoms and time of diagnosis, the number of ACR criteria for SLE, fetal loss, and venous and arterial thrombosis were obtained from medical records. Radiographs of hands and feet were reviewed according to a standard data form that included such details as (sub)luxation, joint space narrowing, metacarpal hook formation, and erosive changes.

The following serological studies were performed in all cases; antinuclear antibody (indirect immunofluorescence on Hep-2 cells), antidsDNA (Farr assay), anti-cardiolipin (enzyme immunoassay), lupus anticoagulant (LAC), anti-ENA (counter immunoelectrophoresis), and rheumatoid factor (Rose-Waaler test).

On the basis of these data a subdivision was made between evident erosive and the nonerosive forms of deforming arthropathy (fig 1). The patients of the non-erosive group were then assessed for the presence of definite Jaccoud's arthropathy, using a "Jaccoud's arthropathy index", which is dependent upon the different clinical symptoms and the severity of the deformities (table 1). The remaining patients were classified as having mild deforming arthropathy.

Because of the small number of patients in the different groups Fisher's exact test was used for statistical analysis.

\section{Results}

Patients with deforming arthropathy could be separated into three groups. The first group consisted of three patients with an erosive form
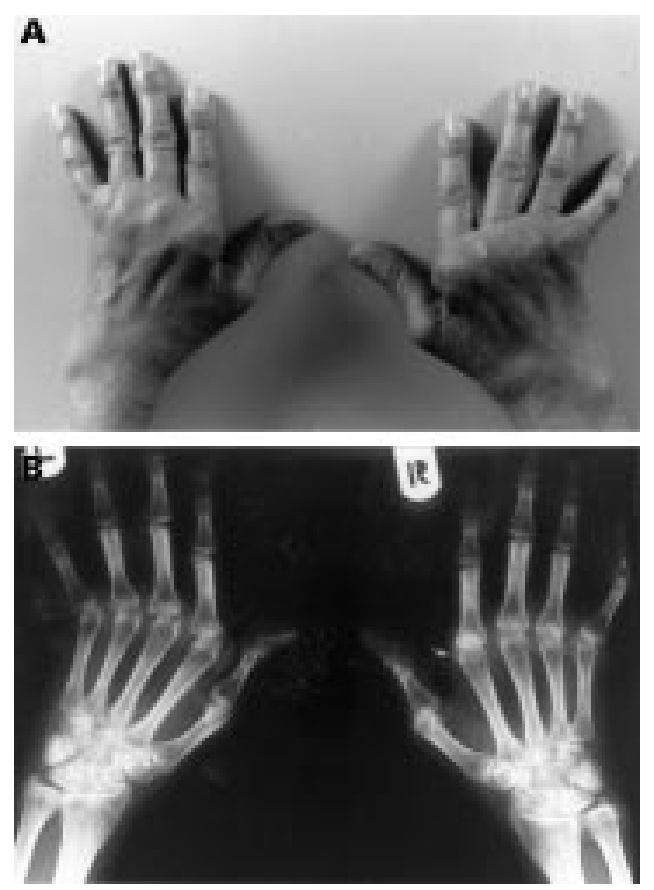

Figure 3 (A) Characteristic joint deviation with MCP subluxation, ulnar deviation, swan neck deformity of the fingers, and $Z$ deformity of both thumbs in faccoud's arthropathy of the hands (lupus hand). (B) Radiograph of same hands showing hook formation (arrow) and alignment disorders without noticeable erosions. 
Table 3 Comparison of ACR criteria in the total SLE population and the three types of deforming arthropathy

\begin{tabular}{lllll}
\hline & $\begin{array}{l}\text { faccoud's } \\
\text { arthropathy } \\
(n=8)\end{array}$ & $\begin{array}{l}\text { Erosive } \\
\text { arthropathy } \\
(n=3)\end{array}$ & $\begin{array}{l}\text { Mild } \\
\text { deforming } \\
\text { arthropathy } \\
(n=6)\end{array}$ & $\begin{array}{l}\text { Non-deforming } \\
\text { arthropathy } \\
(n=159)\end{array}$ \\
\hline Malar rash & $2 / 8$ & $2 / 3$ & $5 / 6$ & $99 / 159$ \\
Discoid rash & $1 / 8$ & $1 / 3$ & $3 / 6$ & $33 / 159$ \\
Photosensitivity & $1 / 8$ & $2 / 3$ & $4 / 6$ & $74 / 159$ \\
Oral ulcers & 0 & $1 / 3$ & $3 / 6$ & $32 / 159$ \\
Arthritis & $8 / 8$ & $3 / 3$ & $6 / 6$ & $128 / 159$ \\
Serositis & 0 & $1 / 3$ & $3 / 6$ & $49 / 159$ \\
Renal disorder & $1 / 8$ & $2 / 3$ & $2 / 6$ & $90 / 159$ \\
Neuropsychological disorder & $1 / 8$ & 0 & $1 / 6$ & $15 / 159$ \\
Haematological disease & $6 / 8$ & $2 / 3$ & $2 / 6$ & $123 / 159$ \\
Anti-nuclear antibody & $8 / 8$ & $3 / 3$ & $6 / 6$ & $156 / 159$ \\
Immunological disorder & $5 / 8$ & $3 / 3$ & $6 / 6$ & $143 / 159$ \\
\hline
\end{tabular}

Table 4 Comparison of laboratory data and thrombotic history of the three types of deforming arthropathy and non-deforming arthropathy

\begin{tabular}{lcccl}
\hline & $\begin{array}{l}\text { faccoud's } \\
\text { arthropathy } \\
(n=8)\end{array}$ & $\begin{array}{l}\text { Erosive } \\
\text { arthropathy } \\
(n=3)\end{array}$ & $\begin{array}{l}\text { Mild deforming } \\
\text { arthropathy } \\
(n=6)\end{array}$ & $\begin{array}{l}\text { Non- } \\
\text { deforming } \\
\text { arthropathy } \\
(n=159)\end{array}$ \\
\hline Livedo reticularis & $4 / 8$ & 0 & 0 & $47 / 159$ \\
Raynaud's phenomenon & $8 / 8$ & $3 / 3$ & 0 & $81 / 159$ \\
Arterial/venous thrombosis & $5 / 8$ & 0 & $1 / 6$ & $38 / 159$ \\
Fetal loss & $4 / 4 \dagger$ & 0 & 0 & $25 / 66 \dagger$ \\
Rose-Waaler test & 0 & $3 / 3$ & $1 / 6$ & not known \\
LAC & $6 / 8$ & 0 & 0 & $39 / 159$ \\
Anticardiolipin IgG & $7 / 8$ & $1 / 3$ & $2 / 6$ & $73 / 159$ \\
Anticardiolipin IgM & $4 / 8$ & 0 & $1 / 6$ & $49 / 159$ \\
Anti-U1RNP & 0 & 0 & $2 / 6$ & $19 / 135^{\star}$ \\
Anti-Sm & $1 / 8$ & 0 & $2 / 6$ & $28 / 135^{\star}$ \\
Anti-SSA & $1 / 6$ & 0 & $1 / 6$ & $36 / 135^{\star}$ \\
Anti-SSB & 0 & 0 & 0 & $8 / 135^{\star}$ \\
\hline
\end{tabular}

^ENA was only performed in 135 of 159 patients.

†Four of eight and 66 of 159 patients had been pregnant.

Table 5 Comparison of radiological features in the hands of the three groups with deforming arthropathy

\begin{tabular}{lccc}
\hline Spectrum radiological features & $\begin{array}{l}\text { faccoud's } \\
\text { arthropathy }\end{array}$ & $\begin{array}{l}\text { Erosive } \\
\text { arthropathy } \\
(n=3)\end{array}$ & $\begin{array}{l}\text { Mild deforming } \\
\text { arthropathy }(n=6)\end{array}$ \\
\hline Wrist & $1 / 8$ & $2 / 3$ & $1 / 6$ \\
Joint space narrowing & $4 / 8$ & 0 & 0 \\
Bone irregularity & $2 / 8$ & $2 / 3$ & 0 \\
Cystic changes & 0 & $3 / 3$ & 0 \\
Evident erosions & $4 / 8$ & 0 & $1 / 6$ \\
Scapholunate dissociation & $2 / 8$ & $3 / 3$ & 0 \\
Palmar/ulnar luxation & 0 & & 0 \\
Fingers & $2 / 8$ & $1 / 3$ & 0 \\
Joint space narrowing & $2 / 8$ & 0 & 0 \\
Bone irregularity & $2 / 8$ & $1 / 3$ & 0 \\
MCP hooks & 0 & $2 / 3$ & $1 / 6$ \\
Cystic changes & & $2 / 3$ & 0 \\
Evident erosions & $4 / 8$ & 0 & \\
MCP (sub)luxation & $4 / 8$ & & \\
Less than 5 & & & 0 \\
More than 5 & & & \\
\hline
\end{tabular}

of deforming arthropathy such as that seen in frank RA (rhupus hand) (fig 2). Using the modified index described by Spronk ${ }^{7}$ we identified eight patients with Jaccoud's arthropathy (or so called lupus hand). This group is characterised by severe deformation (ulnar deviation, swan neck deformities, and $\mathrm{Z}$ deformity of the thumb) of the hands with multiple non-erosive subluxations, mild aching, and little or no evidence of synovitis (fig 3). Peripheral arthritis with mild deformity was seen in the remaining six patients. Table 2 gives the characteristics of the patient cohort. One third of patients with deforming arthropathy were receiving oral corticosteroid treatment (dose ranged from 5 to $20 \mathrm{mg} / \mathrm{d}$, average 7.5) while a further $30 \%$ had received such treatment in the past.

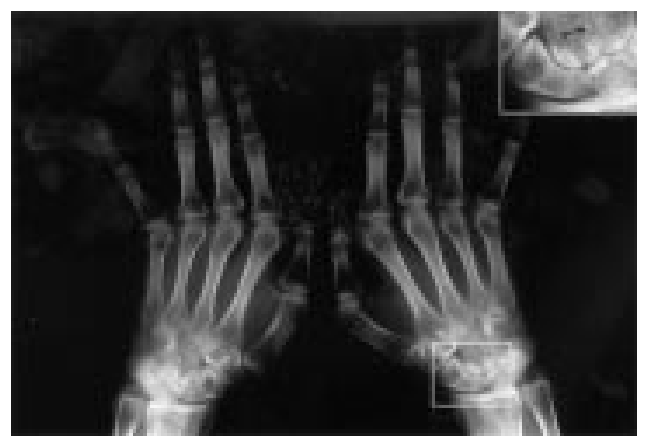

Figure 4 Radiograph of male patient with faccoud's arthropathy showing scapholunate dissociation leading to carpal collapse and mild ulnar translocation of the wrist.

All patients, but one, of the Jaccoud's arthropathy group fulfilled just four criteria of the ACR classification (table 3) and joint symptoms were always found to precede the diagnosis of SLE (mean 13 months, range 6-36 months). Five of them had a history of arterial or venous thrombosis and four of them had had a fetal loss. Renal disorder was the only ACR criterion significantly lower $(p=0.02)$ and fetal loss significantly higher (four of four and 25 of 66 patients who had been pregnant respectively, $p=0.022$ ) in patients with Jaccoud's arthropathy group compared with patients without deforming arthropathy.

Table 4 gives the laboratory profiles and the history of thrombosis. Seven of the eight patients with Jaccoud's arthropathy were positive for anticardiolipin (IgG positive in seven cases and $\operatorname{IgM}$ positive in four). In six of the seven patients positive for IgG anticardiolipin LAC was also present. In the other two groups only three patients were positive for IgG, one of them also for IgM anticardiolipin. The prevalence of LAC and IgG anticardiolipin was significantly higher in patients with Jaccoud's arthropathy compared with patients without deforming arthropathy (seven of eight patients and 73 of 159 patients, $p=0.028$ ). All patients with joint erosions were positive for rheumatoid factor. In the other two groups radiological changes were minimal (table 5).

Irregularity of bone (quite unlike that in RA) was seen in five of the eight patients with Jaccoud's arthropathy. Two of them with marked subluxation showed hook formation of the MCP joints. Four patients showed scapholunate dissociation (fig 4). All patients with Jaccoud's arthropathy had some kind of deformity of the feet (hallux valgus, hammer toes and/or subluxation of metatarsophalangeal joints).

\section{Discussion}

Bywaters was the first to point out the similarity between the deforming arthropathy of SLE and that originally described in 1869 by Jaccoud $^{6}$ for recurrent rheumatic fever; he reported an incidence of five per cent. The clinical picture of Jaccoud's syndrome is characterised by chronic non-erosive deformities (subluxation of the MCP joints, ulnar deviation, which is correctable in the early stages, hyperextension at the proximal interphalangeal joints) that superficially resemble 
those of RA. ${ }^{6}$ Furthermore Bywaters described the formation of "hooks" and cysts of the metacarpal heads in later stages of the disease, which he interpreted as being the result of remodelling of bones to accommodate altered stress. ${ }^{4}$ These same characteristics were used by Spronk et al to develop their "Jaccoud's arthropathy index" to ensure a standard approach in their study. ${ }^{7}$ Alarcon-Segovia et $a \bar{P}$ tried to differentiate the deforming arthropathy of the hands in SLE from that in RA. They considered any deviation, correctible or not, from any of the normal metacarpus finger axes to be deforming arthropathy in SLE. They selected 41 (of 858) SLE patients who had clinical deforming arthropathy and observed swan neck deformities, $Z$ deformity of the thumb, non-erosive MCP joint subluxation, and ulnar deviation. This group of patients also included patients with scant and asymmetric joint erosions. When we used AlarconSegovia's criterion to define patients with deforming arthropathy, subdivided this group into those with an evident erosive and those with a non-erosive form and then applied "Jaccoud's arthropathy index" to the non-erosive group, we identified three forms of deforming arthropathy (Jaccoud's arthropathy, erosive arthropathy, and mild deforming arthropathy).

Arthritis associated with SLE is usually nonerosive, but in a small subset of patients an erosive disease resembling RA develops. We identified erosive arthropathy of the hands (rhupus hand) in three patients (1\%), which is similar to the $1-2 \%$ reported by Dubois. ${ }^{2}$ Whether these patients represent a subset of SLE arthritis or the true coexistence of the two diseases is not clear, but there probably is a real overlap. The serological overlap between RA and SLE is well known with up to 20 per cent of RA patients exhibiting positive antinuclear antibodies (ANA). However, patients with concomitance of RA and SLE are rare, as there are only a small number of well documented cases in literature. ${ }^{1}$

Whereas in RA loss of bone and joint stability are secondary to hypertrophic synovitis, Jaccoud's arthropathy seems to involve mainly ligaments and periarticular soft tissue. Based on histological findings, such as mild but typical fibrous synovitis with little or no round cell infiltration and microvascular changes, Bywaters and others ${ }^{46}$ proposed a prolonged or recurrent low grade inflammatory activity in both the synovial membrane and the capsule as the underlying mechanism. The deformity seen in Jaccoud's arthropathy seems to be the consequence of the loss of ligamentous constraint (resulting from subsequent capsular periarticular fibrosis or synovial vasculitis) in combination with the compressive forces of the muscles acting across the hand rather than the destructive effect of synovitis. The irregularity of bone, which is quite unlike the erosions seen in $R A$, is thought to be attributable to friction rub caused by overlying inflamed tendons or capsule and the altered mechanical forces resulting from subluxation. The close anatomical relation between the extensor carpi ulnaris tendon (as part of the ulnocarpal complex) and the ulnar styloid may explain the high prevalence of erosive damage to this bone mentioned in the studies of Alarcon-Segovia ${ }^{5}$ and Reilly. ${ }^{3}$ Inflammation of the tendon probably plays a major part in the pathogenesis of sudden tendon rupture, which is uncommon in SLE but has been described in several case reports. ${ }^{8}$

Another characteristic of deforming arthropathy observed and used as criterion by Alarcon-Segovia was non-erosive carpal collapse. ${ }^{5}$ In RA hypertrophic synovitis is responsible for disruption of the ligamentous sling within the wrist, producing the joint changes that lead to carpal collapse and subluxation of the joints. ${ }^{9}$ In four patients with Jaccoud's arthropathy we found scapholunate dissociation and rotation of the scaphoid itself that had to be the consequence of weakening or slackening of the (radio-)carpal ligaments. It is interesting that Bywaters ${ }^{4}$ had already noted the gross subluxation of the wrist and radiographs printed in several publications ${ }^{10} 11$ showed evident, scapholunate luxation or ulnar translocation of the wrist that was not described. For patients with Jaccoud's arthropathy the estimated prevalence of articular hypermobility lies between 7 and 50 per cent, depending upon the method of evaluation used. ${ }^{12}$ Although a role for hyperextensibility in the development of Jaccoud's arthropathy seems plausible we found no association.

Jaccoud's arthropathy seems to be a generalised pathological capsular and periarticular condition involving all joints. Although most reports have focused on the hands, Morley et $a l^{13}$ reported three cases of deforming arthropathy of the feet in SLE and coined the term "lupus foot". In accordance with Mizutani and Reilly $^{3}$ we also found a significant association with deformities of the feet (hallux valgus and/or subluxation of metatarsophalangeal joints) in patients with Jaccoud's arthropathy.

Given the wide variety of clinical features associated with SLE, there have been many attempts to identify subsets of patients for whom a given antibody specificity can be identified with deforming arthropathy. Several associations, such as the presence of antibodies against U1 RNP, ${ }^{3}$ RA $33,{ }^{14}$ SS-A/Ro and $\mathrm{SS}-\mathrm{B} / \mathrm{La},{ }^{15}$ have been reported previously. We noted that the patients in our group with Jaccoud's arthropathy represent a subset of SLE. Although all of these patients had clinical features or serological abnormalities suggestive of SLE (mainly arthritis, ANA+, leuco/ thrombocytopenia, rash) it took several years to satisfy just four of the ACR classification criteria for the diagnosis of SLE. Previously these patients were considered as having a lupus-like syndrome. Furthermore in our study there seems to be a remarkable association with fetal loss, thrombosis-both venous and arterialand the presence of antiphospholipid antibodies. Our data suggest a striking coexistance of Jaccoud's and the antiphospholipid syndrome ${ }^{16}$ although we cannot explain the pathogenetic link. It is possible that small vessel vasculopathy plays a part in the genesis of the periarticular fibrosis. Some evidence for this hypothesis could be found in "fibrin like material 
obliterating small vessel lumens" described in certain synovial biopsy specimens. ${ }^{2}$ Bywaters already reported a correlation with mitral stenosis and Libman-Sacks endocarditis, ${ }^{4}{ }^{17}$ both said to be associated with antiphospholipid syndrome. Sturgess et $a l^{18}$ and Palazzo et $a l^{19}$ described patients with hypocomplementaemic urticarial vasculitis syndrome and the combination of Jaccoud's syndrome, valvulopathy and vasculopathy of the small cutaneous vessels and the femoral and renal arteries.

The group with mild deforming arthropathy did not differ in any respect from the SLE population without deforming arthropathy.

In conclusion, allowing for the limitations of a definition of deforming arthropathy, we have presented some clinical guidelines for subdividing deforming arthropathy into several clinical forms that are relevant in clinical practice for early diagnosis and the differential diagnosis of SLE (for instance, distinguishing it from RA).

Patients with Jaccoud's arthropathy were characterised by a slow evolution into classifiable SLE, distinct radiological features, association with foot involvement and association with the presence of lupus anticoagulant and anticardiolipin antibodies. We believe that these guidelines will facilitate the clinical management of deforming arthropathy.

In conclusion we have presented some guidelines that facilitate the division of lupus related deforming arthropathy, into distinct subgroups with differing clinical and serological characteristics. Patients with Jaccoud's arthropathy are notable for the slow evolution of their disease into classifiable SLE, unique radiological features, foot involvement and the association with lupus anticoagulant and anticardiolipin antibodies, while those with a "mild deforming arthropathy" do not seem to differ in any respect from SLE patients without arthropathy. We believe that defining lupus arthropathy in the manner described will assist in clinical management, from the time of diag- nosis, for example, in distinguishing RA from SLE, through treatment dicisions based on likely progression.

1 Maddison PJ, Isenberg DA, Woo P, Glass DN. Oxford textbook of rheumatology. Oxford: Oxford University Press 1998;5.7:1147-8

2 Wallace DJ, Hahn BH, Quismorio FP, Klinenberg JR. Dubois' lupus erythematosus. 5th ed. Philadelphia: Lea and Febiger, 1997:635-9.

3 Reilly PA, Evision G, McHugh NJ, Maddison PJ. Arthropathy of hands and feet in systematic lupus erythematosus. $\mathrm{J}$ Rheumatol 1990;17:777-84.

4 Bywaters EGL. Jaccoud's syndrome. Clin Rheum Dis 1975; $1: 125-48$.

5 Alarcon-Segovia D, Abud-Mendoza C, Diaz-Jouanen E, Iglesias A, De Los Reyes V, Hernández-Ortiz J. Deforming arthropathy of the hands in systemic lupus erythematosus. arthropathy of the hands in

6 Jaccoud FS. Sur une forme de rhumatisme chronique. Lecons de clinique Médicale faites a l'Hopital de la Charité. Paris: Delaclinique Médicale faites

7 Spronk PE, Ter Borg EJ, Kallenberg CGM. Jaccoud arthropathy; a clinical subset with an increased C-reactive protein response? Ann Rheum Dis 1992;51:358-61.

8 Kahn MA, Ballou SP. Tendon rupture in systemic lupus erythematosus. J Rheumatol 1981;8:308-10.

9 Taleisnik J. The wrist. New York: Churchill Livingstone, 1985:357-63.

10 Sukenik S, Hendler N, Yerushalmi B, Buskila D, Liberman N. Jaccoud's-type arthropathy: an association with sarcoidosis. J Rheumatol 1991;18:915-17.

11 Szczepanski L, Targonska B, Piotrowski M. Deforming arthropathy and Jaccoud's syndrome in patients with systemic lupus erythematosus. Scand J Rheumatol 1992; 21:308-9.

12 Babini SM, Cocco M, De la Sota M, Babini JC, Arturi A, Marcon JC, et al. Tendinous laxity and Jaccoud's syndrome in patients with systematic lupus erythematosus. Possible role of secondary hyperparathyroidism. J Rheumatol 1989; 16:494-8.

13 Morley KD, Leung A, Rynes RI. Lupus foot. BMJ 1982;284:557-8.

14 Isenberg DA, Steiner G, Smolen JS. Clinical utility and serological connections of anti-RA33 antibodies in systemic lupus erythematosus. J Rheumatol 1994;21:7: 1260-3.

15 Franchescini F, Cretti L, Quinzanini M, Rizzini FL, Cattaneo R. Deforming arthropathy of the hands in systemic lupus erythematosus is associated with antibodies to SSA/Ro and to SSB/La. Lupus 1994;3:419-22.

16 Derksen RHWM, de Groot PhG, Kater L, Nieuwenhuis HK. Patients with anti-phospholipid antibodies and venous thrombosis should receive long term anti-coagulant thrombosis should receive long term an
treatment. Ann Rheum Dis 1993;52:689-92.

17 Bywaters EGL. The relation between heart and joint disease (type Jaccoud's). Br Heart J 1950;12:101-31.

18 Sturgess AS, Littlejohn GO. Jaccoud's arthritis and panvasculitis in the hypo-complementemic urticarial vasculitis syndrome. J Rheumatol 1988;15:858-61.

19 Palazzo E, Bourgeois P, Meyer O, De Bandt M, Kazatchkine M, Kahn M. Hypo-complementemic urticarial vasculitis syndrome, Jaccoud's syndrome, valvulopathy: A new syndromic combination. J Rheumatol 1993;20:1236-40. 